\title{
Cientitifíca
}

http://revistas.udistrital.edu.co/ojs/index.php/revcie/index

\section{Estructura de datos XTCE en sistemas CubeSat}

\author{
XTCE Data Structure in CubeSat Systems
}

\section{Estrutura de dados XTCE em sistemas Cubesat}

\author{
Wilmer Figueredo ${ }^{1}$ \\ Lilia Edith Aparicio $\mathrm{PicO}^{2}{ }^{\mathbb{D}}$
}

Recibido: abril de 2020

Aceptado: octubre de 2020

Para citar este artículo: Figueredo, W., Aparicio Pico, L. E. (2021). Estructura de Datos XTCE en Sistemas CubeSat. Revista Científica, 40(1), 102-117. https://doi.org/10.14483/23448350.16132

\section{Resumen}

Este articulo muestra la construcción de un modelo de información para el intercambio de datos e información dentro del contexto del desarrollo de sistemas satelitales de tipo CubeSat. Se articulan el desarrollo, procedimiento, descripción, diseño, arquitectura y los resultados que refieren en gran medida a la adopción de dichos lineamientos en un escenario de interoperabilidad tecnológica. De igual manera, se puntualiza la funcionalidad en los esquemas de trasmisión de información de telemetría en satélites académicos. Se tienen en cuenta los lineamientos del Comité Consultivo para Sistemas de Datos Espaciales (CCSDS), en colaboración con la Agencia Espacial Europea (ESA) y la entidad de Cooperación Europea para la Normalización Espacial (ECSS), las cuales han consolidado múltiples recomendaciones para normalizar la transferencia de información de sistemas espaciales a través de varios procedimientos y mecanismos. Adicionalmente, se consideró el lenguaje de marcado extensivo $(\mathrm{XML})$, junto al formato de intercambio de telemetría y comando (XTCE), en conjunto con el estándar de utilización de paquetes (PUS), la descripción de los servicios de operación de misión (MO) y la capa de abstracción de mensajes (MAL), teniendo en cuenta que sugieren la modularidad y especificidad necesaria para el desarrollo y construcción de sistemas de información en tecnologías satelitales. Palabras clave: gestión de datos, medición, satélite, telemando, telemetría, XML.

\begin{abstract}
This article shows the construction of an information model for the exchange of data and information within the context of the development of CubeSat type satellite systems; articulating the development, procedure, description, design, architecture and results that largely refer to the adoption of such guidelines within a scenario of technological interoperability and specifying the functionality in the transmission patterns of telemetry information in academic satellites. The guidelines of the Consultative Committee on Spatial Data Systems (CCSDS) are taken into account in collaboration with the European
\end{abstract}

1. Axede S.A. Bogotá, Colombia. wilmerfr@gmail.com

2. Universidad Distrital Francisco José de Caldas. Bogotá, Colombia. medicina@udistrital.edu.co 
Space Agency (ESA) and the European Cooperation for Spatial Standardization (ECSS), who have consolidated multiple recommendations to standardize the transfer of information from space systems through various procedures and mechanisms. Additionally, the Extensive Markup Language (XML) was considered, next to the Telemetry and Command Exchange (XTCE) format, in conjunction with the Package Utilization Standard (PUS), the description of Mission Operation Services (MO) and the Message Abstraction (MAL) layer, taking into account that they suggest the modularity and specificity necessary for the development and construction of information systems in satellite technologies.

Keywords: command, data management, measurement, satellite, telemetry, XML.

\section{Resumo}

Este artigo mostra a construção de um modelo de informação para o intercâmbio de dados e informações no contexto do desenvolvimento de sistemas de satélite tipo Cubesat; articulando o desenvolvimento, procedimento, descrição, design, arquitectura e resultados que se referem em grande medida à adopção de tais orientações num cenário de interoperabilidade tecnológica e especificando a funcionalidade nos padrões de transmissão de informações de telemetria em satélites académicos. As orientações do Comité Consultivo para os Sistemas de Dados Espaciais (CCSDS) são tidas em conta em colaboração com a Agência Espacial Europeia (ESA) e a Cooperação Europeia para a Normalização Espacial (ECSS) que consolidaram múltiplas recomendações para padronizar a transferência de informação dos sistemas espaciais através de vários procedimentos e mecanismos. Além disso, foi considerada a Linguagem de Marcação Extensiva (XML), ao lado do formato de Intercâmbio de Telemetria e Comando (XTCE), em conjunto com o Padrão de Utilização de Pacotes (PUS), a descrição dos Serviços de Operação de Missão (MO) e a camada de Abstracção de Mensagens (MAL), tendo em conta que sugerem a modularidade e especificidade necessárias para o desenvolvimento e construção de sistemas de informação em tecnologias de satélite.

Palavras-chaves: comando, gestão de dados, medição, satélite, telemetria, XML.

\section{Introducción}

Independiente de la misión, finalidad u objetivo con el cual un satélite académico de tipo CubeSat es construido, sus fases fundamentales para la puesta en órbita no difieren de satélites comerciales, militares, de investigación o de experimentación. Sin embargo, limitaciones que corresponden a características constructivas, recursos de operación, esquemas de comunicación, la órbita de operación y hasta el tiempo de vida útil sugieren un gran reto interdisciplinar en las áreas de desarrollo e investigación enfocadas en la mejora continua de la tecnología aeroespacial de bajo costo (Crisp et al., 2015).

Una vez un satélite CubeSat es puesto dentro del espacio orbital para el cual fue diseñado, este entra en varias etapas de estabilización y operación acordes a su misión (Vertat et al., 2018). Durante dichas etapas, y particularmente durante la fase de operación normalizada, los sistemas de telemetría y mando a distancia del satélite, en conjunto con los mismos sistemas del nodo en tierra, juegan un papel importante durante la vida útil del satélite (Díaz y Hernández, 2015). El proceso de intercambio de información para conocer el estado general de los sistemas, módulos, subsistemas, componentes y comportamiento en general del sistema en órbita permite a los operadores en tierra tomar acciones sobre la operación normal y, en casos particulares, acciones tempranas, proactivas y hasta reactivas para garantizar la continuidad operativa de un satélite.

No obstante, la operación de sistemas satelitales CubeSat de carácter académico conlleva a correlacionar características que a su vez involucran a los nodos de comunicación en órbita y en tierra, tales como: las tasas de transferencia, la calidad del canal, el índice de información trasferida y otras tantas, que conciernen directamente en la calidad, eficiencia y efectividad de la comunicación entre un nodo y otro (Ravichandran et al., 2009). Estas características se ven condicionadas a: la órbita que el satélite describe, generalmente 
esta se limita a una órbita baja terrestre (LEO), particularidad que atribuye limitantes funcionales al sistema en general y a los operadores del sistema en tierra (Fernandez et al., 2017). En segundo lugar, la baja utilidad operativa y funcional dado el periodo orbital y reducidos intervalos de eclipse entre un CubeSat y la estación o estaciones del mismo proyecto.

Adicional a lo anterior, las redes de estaciones terrenas para sistemas CubeSat y, en particular, las redes de estaciones terrenas con fines de investigación y experimentación académica (Berthoud y Schenk, 2016) se han diseñado y configurado como una herramienta colaborativa que mitiga de una u otra forma los efectos del comportamiento y mecánica orbital dada la naturaleza constructiva de estos sistemas. Además, tienen por objetivo fundamental consolidar la interconexión de estaciones terrenas que comparten características similares o equivalentes para la habilitación de canales de comunicación; ampliando así los intervalos de transferencia de información efectiva de un satélite en órbita (Díaz González et al., 2016).

En este sentido, han surgido como una alternativa tecnológica que facilita recursos y servicios de conectividad a un satélite a través de la disponibilidad global de otras estaciones terrenas tecnológicamente compatibles (Cappiello et al., 2019), como se muestra en la figura 1. El diagrama en bloques por niveles de esta imagen modela dos sistemas satelitales y una estación terrena, desde la cual es posible recibir y transferir información mediante los servicios de comunicación atravesando los diferentes módulos de telemetría y comando respectivos hasta llegar a la capa de aplicaciones. Es importante tener en cuenta que los satelitales de carácter académico y de investigación determinan ciertas características propietarias en los productos de desarrollo que, a su vez, generan incompatibilidad tecnológica entre un satélite y un grupo de estaciones terrenas, por lo que las iniciativas de interconexión y recursos compartidos de las redes de estaciones terrenas se ven limitadas (Espindola, 2017).

La participación en redes de estaciones terrenas de carácter académico y de investigación tales como SatNet (Espindola, 2017), SatNogs (White et al., 2015), QB50 (Kilic et al., 2013), entre otras, puede ser abordadas de dos formas. La primera: participación abierta y segura, empleando tecnologías estandarizadas en sus procesos de comunicación, operación y funcionamiento que permita

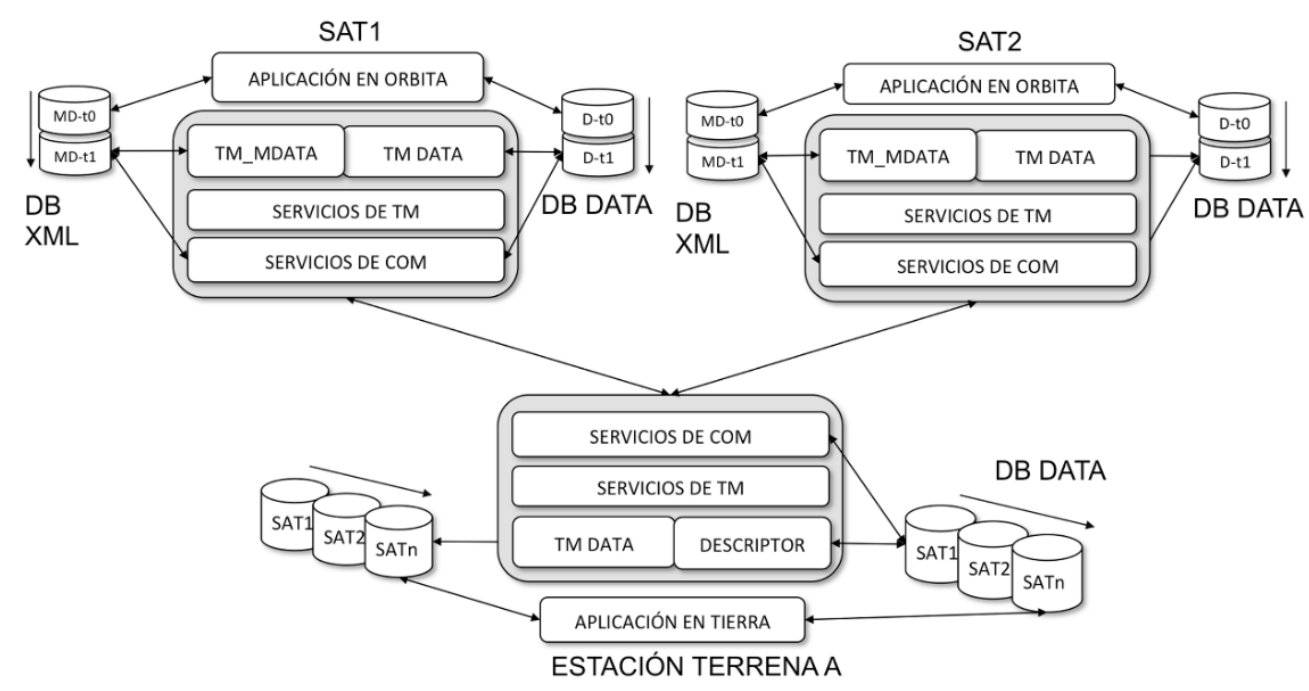

Figura 1. Diagrama de Bloques comunicación satelital. Fuente: adaptación de Shamutally (et al., 2018). 
alinear tecnologías entre múltiples proyectos. La segunda: participación restrictiva y limitada, la cual emplea desarrollos de sistemas de información y comunicación propietarios. En consecuencia, las metodologías actuales de desarrollo tecnológico para sistemas satelitales CubeSat limitan el desarrollo colaborativo eficiente en la investigación técnica y científica de esta tecnología que tiene por finalidad la optimización y mejora continua de los sistemas y esquemas de información y comunicaciones, apalancados en tecnologías accesibles y limitadas en recursos (Shahu, 2018).

\section{Metodología}

Durante la etapa de diseño de sistemas CubeSat uno de los módulos que debe ser considerado como objeto fundamental dentro de cualquier arquitectura es el subsistema de telemetría. En este módulo se realiza la medición de variables físicas (voltajes, corrientes, temperaturas, presión, niveles, etc.), comportamiento (activo, inactivo, cerrado, abierto, apagado, encendido, etc.), incluso indica si la información de carga útil de un satélite consiste en la medición de variables astronómicas (Moreno y Sánchez, 2014). Este sistema da a los operadores y usuarios del sistema en tierra la información elemental y esencial para determinar, monitorear, controlar y operar el funcionamiento del satélite durante su vida útil.

La información de telemetría de un sistema satelital de tipo CubeSat es generada por los sensores que se distribuyen a lo largo de la infraestructura del satélite y fuentes de información lógica de uno o varios subsistemas o dispositivos del mismo satélite (Electronics, Computing and Communication Technologies, 2014). Durante las fases de estabilización, posterior fase de operación y de manera previa a estas dos, los desarrolladores de sistemas aeroespaciales emplean diferentes esquemas de lenguaje de datos para codificar, empaquetar y distribuir la información de telemetría. Del mismo modo, emplean diversas tecnologías para consolidar los sistemas de transferencia que pueden ser producto de índole propietaria, estandarizada $y$, en otros casos, productos de un estándar modificado con el fin de estructurar, codificar y almacenar de manera temporal para luego transmitir la datos de telemetría desde el satélite hasta la tierra a través de canales de radio sobre los cuales se transportan información sobre campos de datos, tramas o paquetes de información asociados a uno o varios protocolos de comunicación (Moreno y Sánchez, 2014).

Una vez la información de telemetría es colectada por los sensores del satélite, esta puede ser almacenada o procesada por el On Board Computer o computador a bordo (OBC) del satélite para luego ser transferida desde el sistema en órbita hacia diferentes estaciones en tierra mediante uno o varios sistemas de comunicación construidos según los diseños y requerimientos particulares de un proyecto (Eatchel et al., 2015), los cuales emplean diferentes mecanismos de modulación, codificación, protocolos y niveles de potencia haciendo que la recepción de información sea una labor exigente a la hora de preparar un sistema de comunicaciones en tierra (Alhammadi y Svetinovic, 2017).

De igual forma, la interpretación y utilidad de la información recibida por una estación terrena no presenta un aspecto ni comportamiento lineales en todos los desarrollos (Scholz y Juang, 2015). En consecuencia, las condiciones de conexión, la calidad de esta, la agilidad en los procesos de actualización, la utilidad del sistema de información y el posterior acceso a la información recibida por parte de terceras estaciones terrenas (en el mejor de los casos) convierte los procesos de colaboración en el ámbito de desarrollo de proyectos aeroespaciales en una tarea compleja en términos operativos y de diseño para un sistema satelital.

Es importante resaltar que la información de telemetría puede o no ser recibida, procesada, interpretada y utilizada por un nodo de comunicaciones en tierra por los efectos de la heterogeneidad de los protocolos utilizados, las estructuras de datos y los mecanismos de comunicación empleados por 
diferentes misiones espaciales (Praks Jaan, 2016). Con el fin de generar sinergia operacional, funcional y un entorno colaborativo consistente, además de una mayor participación entre proyectos satelitales de carácter académico, es necesario diseñar una propuesta basada en una serie de pautas y reglas que proporcionen, en primera medida, un sistema de información predictivo, un esquema de asociación y, posteriormente, reglas de comunicación dentro de un amplio conjunto de posibilidades que garanticen la transferencia de información de telemetría de un satélite en órbita a un grupo o red de estaciones terrenas con el fin de lograr el intercambio de información y colaboración de todos los participantes de manera ágil, propicia y abierta (Felt, 2014).

\section{Formato de datos}

El formato de intercambio de telemetría y comando (XTCE) (Simon et al., 2006) es una recomendación de carácter abierto publicado por el Comité Consultivo para Sistemas de Datos Espaciales (CCSDS) para el intercambio de información y bases de datos de telemetría y comando entre los sistemas que se ven involucrados en la comunicación y el intercambio de información satelital (Mision Operations Reference Model 1, 2010). Este modelo es un esquema genérico de alto nivel que puede contener tanta información como sea posible acerca de un sistema mismo.

En términos prácticos, las características fundamentales de una base de datos estándar no difieren de una base de datos XTCE. Pues estas cuentan con una estructura de datos definida que parte de la declaración de campos que a su vez contienen registros que, además, poseen una amplia estructura a lo largo de categorías de información y que se distribuyen en un intervalo de tiempo (Shruthi et al., 2017). De esto modo, la descripción de un sistema satelital de tipo CubeSat junto con su estado puede ser descrito con parámetros que refieren de manera general o particular a componentes, módulos, subcomponentes, submódulos o dispositivos del sistema como se presenta en la figura 2.

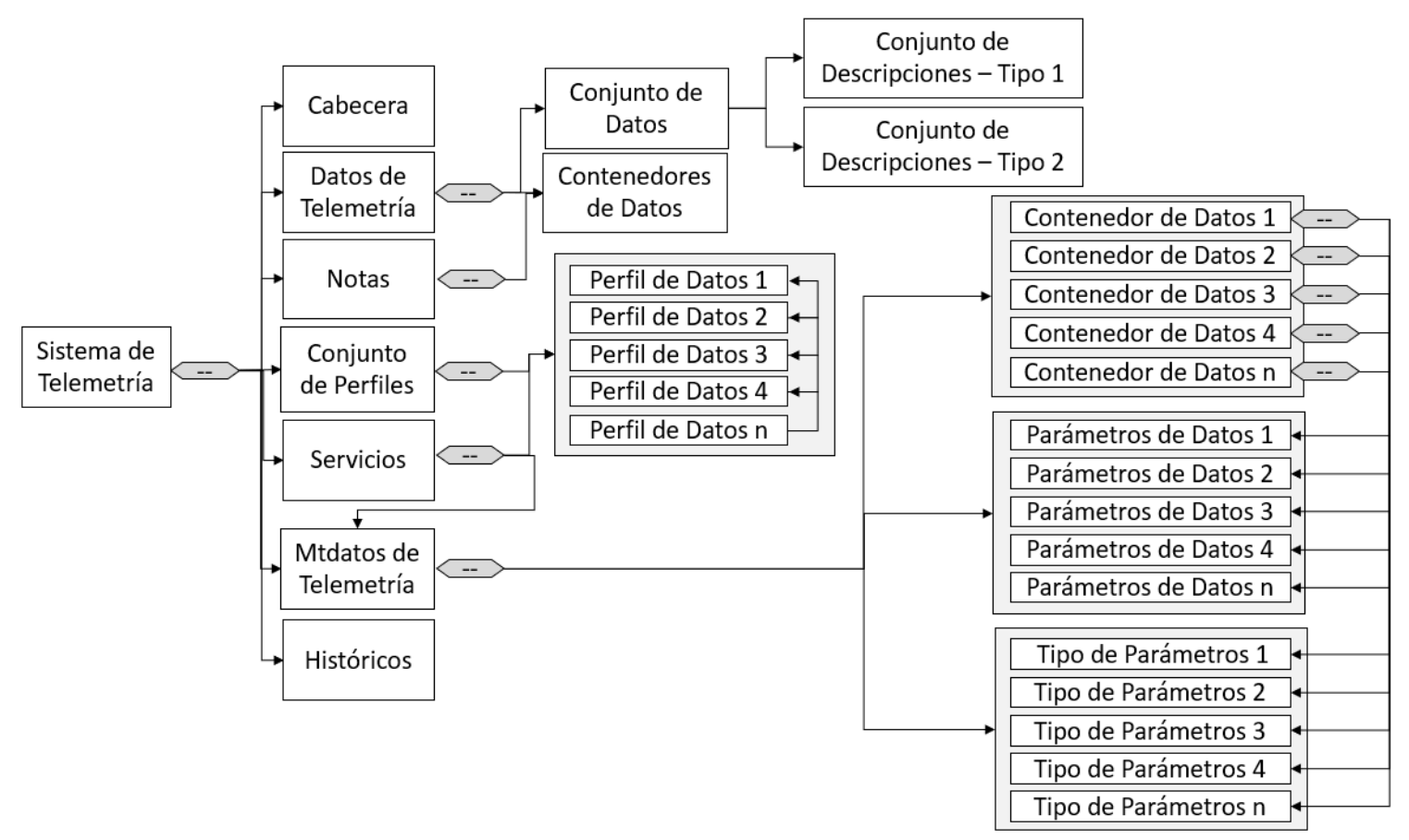

Figura 2. Niveles de distribución de información para la base de datos XTCE.

Fuente: adaptación de Simon et al. (2006). 


\section{Estructura}

La base de datos XTCE está representada por completo en un formato $\mathrm{XML}$, siendo esta la naturaleza propia de la recomendación y quizá la mejor práctica para alojar y utilizar la información dentro del sistema mismo dada la flexibilidad para representar la semántica y sintaxis de la información (Thiruppathirajan et al., 2014). En este orden, un documento XML (el cual realmente es una base de datos) puede contener uno o varios documentos más de tipo XML; así mismo, puede restringir, denegar condicionalmente o proporcionar acceso a cualquier segmento de información contenida en dicho formato. De modo que XML se convierte en la base constructiva del formato XTCE en el cual a través de modificaciones al lenguaje mismo es posible describir y definir todas y cada una de las características (Mishurov et al., 2017) de un sistema de satelital, tales como los módulos, submódulos, dispositivos, unidades de medidas, magnitudes, valores máximos, mínimos, constantes, etc.

Las características de una base de datos XTCE parten del concepto de jerarquías de información dentro del cual se estructura y describe el sistema satelital CubeSat y las acciones que este debe ejecutar (Anandan et al., 2018). Dichas estructuras de datos pueden corresponder al satélite propiamente, a la información u operaciones asociadas a un componente particular o general, a la notificación de información al comportamiento que debe ejecutar una constelación de satélites, a un grupo de estaciones terrenas, a un proyecto satelital o la información relacionada con los mismos sensores. Por esta razón, es preciso acotar que dentro de la estructura de base de datos XTCE existen dos categorías las cuales pueden ser manipuladas como bases de datos conjuntas o individuales (Miyazaki et al., 2020). Esto es: una base de datos y estructura de datos de información de telemetría y una segunda base de datos de control del satélite en órbita.

De otra parte, la base de datos se ha segmentado en contenedores de información que empaquetan la datos descriptivos e identificadores de uno o varios componentes de interés. Por tanto, la información perteneciente a un determinado contenedor se ha parametrizado con nombres de variables y magnitudes, los cuales pueden tener connotación discreta o continua dentro del límite natural del sistema o dispositivo mismo (Vera-Cely et al., 2015). Una vez definidos los parámetros de información es preciso asociar también parámetros de codificación, decodificación, algoritmos y sincronización que emplean los datos para representar dicha información para de esta forma proporcionar el nivel de completitud a la información.

Cabe resaltar que el formato XTCE, además de ser una base de datos de información para el sistema de telemetría y telemando, también puede contener cualquier otro tipo de datos orientados a propósitos particulares tales como: información asociada a servicios de comunicación de capa superior, mensajes de servicio para niveles subyacentes e información relacionada con la misión o carga útil del satélite (Simon et al., 2006).

Es posible considerar de manera abstracta esta estructurara de información como un esquema de información que puede dotar al sistema satelital (satélite y estación terrena) con un conjunto de servicios y mensajes que apoyan la construcción de un modelo formal en capas del protocolo de telemetría en un entorno de comunicación espacio a tierra como el que se presenta en la figura 3.

En el nivel superior, nivel de aplicación, se ubican todas las herramientas desarrolladas de manera individual y específica a una misión o proyecto espacial. En particular, el sistema de adquisición de información de estado o telemetría en órbita y la interfaz de usuario en tierra. Este primer nivel tiene por objetivo colectar, organizar, y almacenar en una base de datos XTCE las variables numéricas que describen el estado del satélite, así como presentar dicha información en una interfaz gráfica que facilite el análisis de información a través del tiempo, de tal forma que el sistema de telemetría deberá tener capacidades para entregar la información de estado y condición del satélite mediante la estructura de datos definidas de manera previa al 


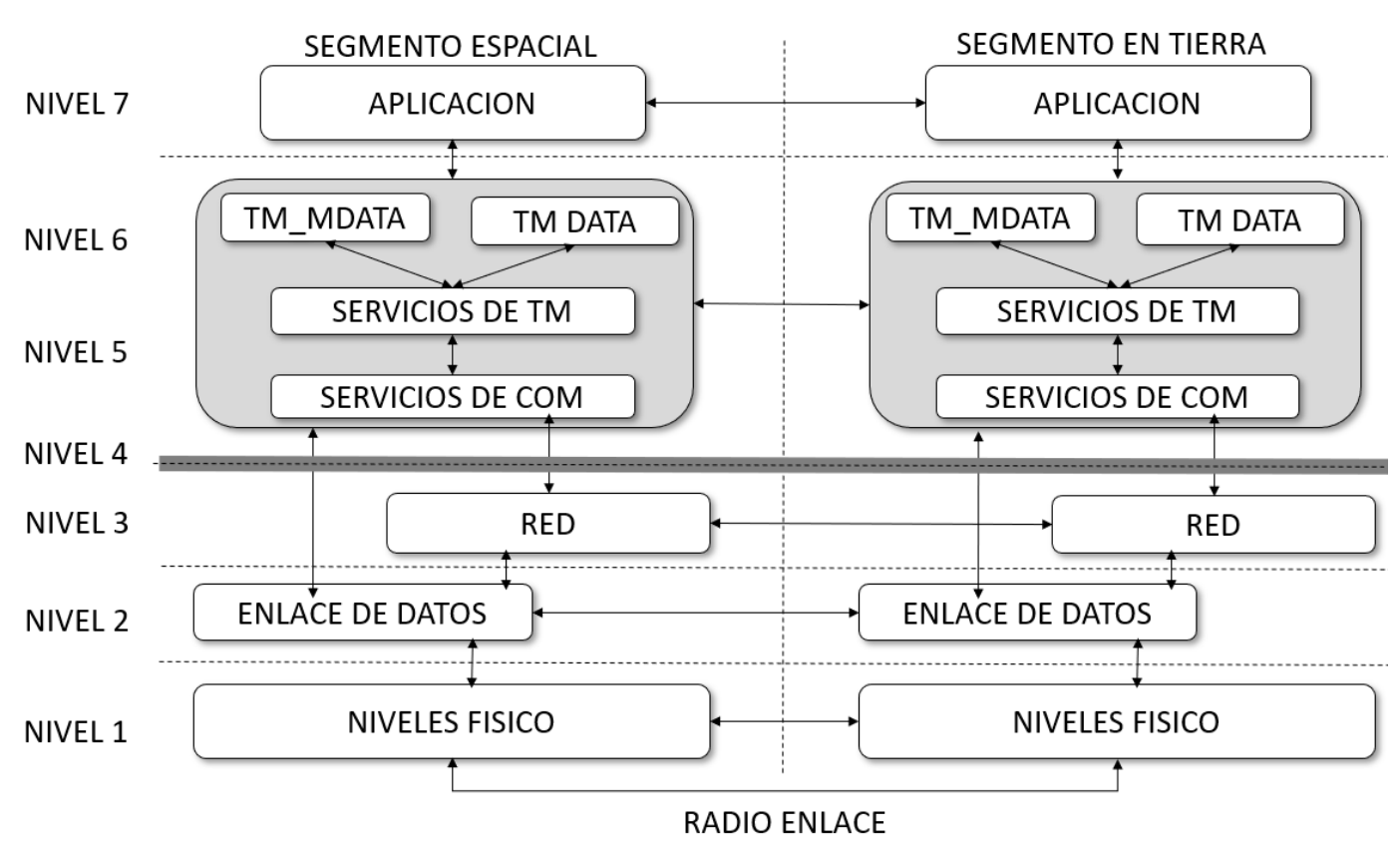

Figura 3. Modelo en capas del sistema de información de telemetría.

Fuente: adaptación de Alhammadi y Svetinovic (2017).

lanzamiento del sistema satelital y acorde a las recomendaciones XTCE de la CCSDS (Mision Operations Reference Model 1, 2010)).

En los niveles siguientes (cinco y seis del mismo modelo) se ubica el sistema lógico para transferencia de datos de telemetría. Este sistema se encuentra subdivido por cuatro entidades que componen al servicio de transferencia:

TM_SCOM (servicio de comunicación de telemetría) es una entidad que ejecuta las funciones de servicio de comunicaciones para el sistema. Además de ser un controlador entre los niveles inferiores y las demás entidades o componentes del sistema de telemetría, actúa como interfaz de control para los recursos del nivel de transporte o los protocolos de comunicación de niveles inferiores como: AX25, CSP, CCSDS-SPP y CCSDS-SDLP y para el mantenimiento lógico de las sesiones de comunicación. Tanto en órbita como en tierra, esta entidad constituye la interfaz de transferencia de datos directa para los niveles uno como el subsistema de radio faro y niveles subyacentes dentro de un sistema de características propietarias. En caso contrario, el sistema en un entorno estandarizado ofrece servicios de comunicación a los niveles de transporte MO, MAL, PUS y nivel físico (radio faro) según las necesidades de cada misión.

El subsistema (servicio de telemetría) TM_SERVICE es una entidad diseñada para el manejo de los servicios de telemetría que asegura la entrega confiable de información entre entidades adyacentes y niveles subyacentes del modelo de comunicaciones. Esta encamina las solicitudes de conexión y desconexión de acuerdo con los requerimientos de transferencia, recepción de información y pérdida de conectividad, con el fin de ejecutar procedimientos simples para facilitar las rutinas de control de procesos y servicios de transferencia y recepción de datos de estado durante una conexión de transferencia. Adicionalmente, actualiza al componente de servicio de conectividad TM_SCOM acerca del estado de una conexión, así como la aceptación de apertura o cierre de una conexión de servicio y validación de los niveles y criterios de acceso a la información de telemetría. 
La entidad de control de datos de telemetría (TM_DATA) es un componente de software que encamina dos tipos de solicitudes: datos y el conjunto de datos y metadatos cuando son requeridos durante una misma solicitud, hacia la base de datos y la entidad TM_SERVICE. Esta entidad también es un servicio de almacenamiento volátil, temporal y recurrente de la información de datos y metadatos de telemetría enviada por la base de datos a la entidad TM_SOM. Esta función permite recuperar mensajes durante conexiones establecidas.

Por su parte, la entidad de control de metadatos de telemetría (TM_MDATA) es un componente de software que encamina las solicitudes de metadatos hacia la base de datos y las respuestas hacía la entidad TM_SERVICE; esta función permite recuperar mensajes durante conexiones establecidas. Este modelo y sus componentes particulares constituyen un sistema de aplicación para la gestión y transferencia eficiente de información de telemetría en proyectos académicos para la construcción y desarrollo de pequeños sistemas satélites.

\section{Resultados}

En la raíz del esquema de datos XTCE se ubica la descripción del sistema satelital, la cual corresponde a la representación de elementos que definen de manera general al sistema satelital y, para este caso particular, también a los componentes de telemetría. Cualquiera que sea la aplicabilidad, la descripción puede estar asociada a un componente del sistema satelital o el satélite en sí. Por ejemplo, dicha descripción puede hacer referencia únicamente a la información de telemetría asociada al módulo de potencia del sistema en órbita. La descripción del sistema incluye el nombre de un proyecto satelital, así como de un grupo de trabajo, sus autores, un equipo de recurso humano en tierra, fechas de creación, edición, datos de clasificación, estado de validación, listas de notas que pueden ser textuales y demás parámetros generales que permitan conocer en síntesis la estructura y objeto del sistema satelital. A su vez, esta definición incluye alternativas para referenciar subsistemas.

Es importante notar en las grandes arquitecturas, en las que un sistema satelital puede contener a otro, las estructuras jerárquicas de asignación de variables, parámetros y categorías que se hacen necesarios con el fin de mantener la usabilidad y herencias de distribución de datos a lo largo de la arquitectura del satélite, de manera similar a la estructura del sistema raíz.

La distribución de la información, en conjunto con la asignación de nombres, es relevante a la hora de evitar colisiones en los dominios de nombres y variables, dado que hay descriptores que redundan a lo largo de la arquitectura del sistema. En la figura 4 se muestra la implementación de la cabecera o el esquema XTCE para la definición del sistema satelital asociado al proyecto CubeSat-UD.

Los sistemas de nombres de cada una de las clases se han considerado como parámetros reservados tal como lo menciona el estándar (21) para mantener la heterogeneidad entre las múltiples aplicaciones y desarrollos particulares. Sin embargo, estos pueden ser editados mediante la manipulación de los esquemas o formatos de información XTCE o XML que, posteriormente, serán verificados a través del desarrollo o edición de compiladores.

La figura 5 muestra la estructura del encabezado XTCE para construcción de la base de datos de telemetría para el proyecto CubeSat UD. en esta se puede observar remarcado el arreglo de apertura y cierre de cada conjunto de información dependiente del encabezado de información. En resumen, la agrupación de datos generales del sistema espacial: conjunto datos auxiliares para la presentación y conjunto de datos de cabecera que corresponden a notas textuales para la descripción del sistema.

Además de los tipos de datos, es necesario referenciar el conjunto nombres con los parámetros correspondientes que definen al dato mismo. La figura 6 muestra un ejemplo de dicha asociación, 


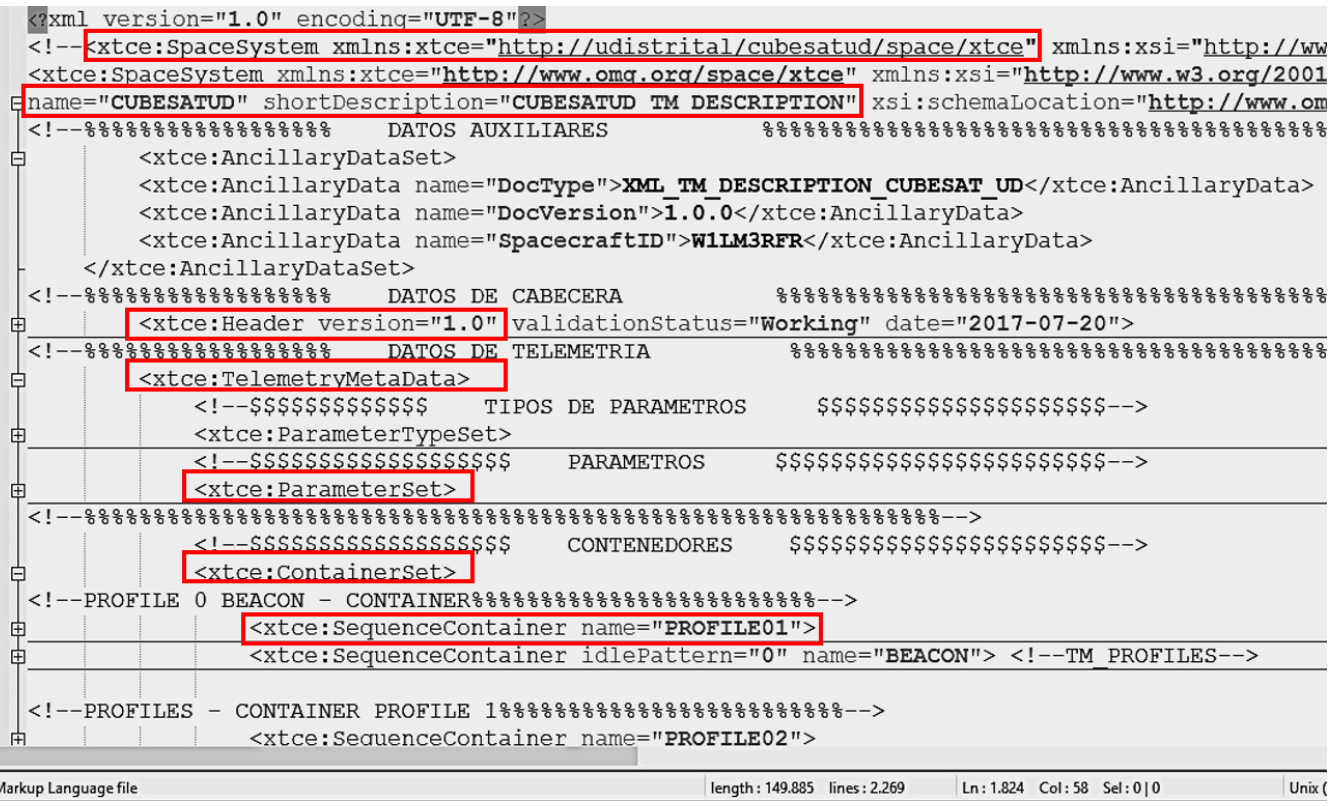

Figura 4. Cabecera y estructura XTCE de la base de datos CubeSat.

Fuente: elaboración propia del autor.

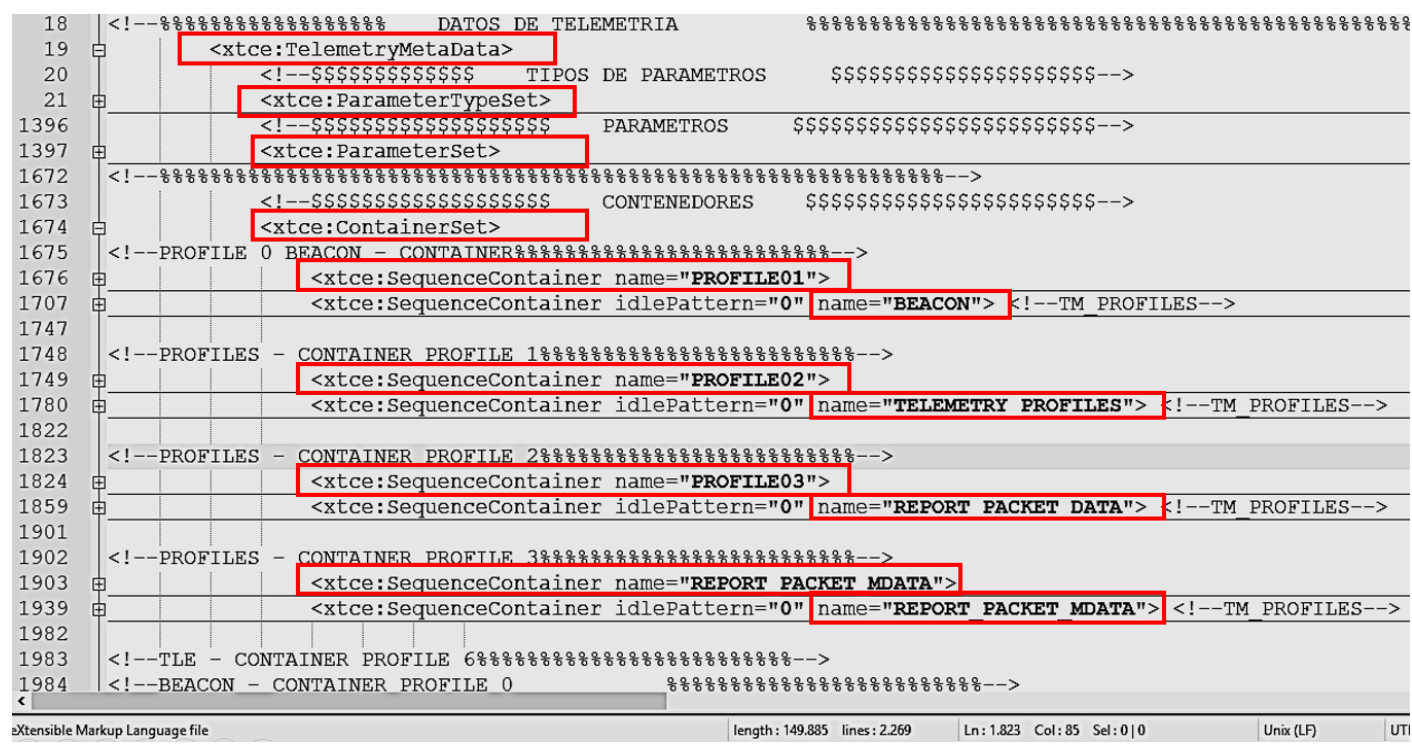

Figura 5. Formato de encabezado XTCE, base de datos CubeSat-UD.

Fuente: elaboración propia del autor.

pues, en la misma figura, se observa la referencia del tipo de parámetro "NameSatType" que corresponde a un tipo de dato con su respectivo valor "NameSat", el cual referencia a un campo de información dentro de trama de telemetría que será enviada por el sistema de radio faro "Beacon".
De esta forma, se ha logrado acotar el conjunto de información que puede estar relacionada con datos propios de telemetría o datos que la describen. Para ambos casos es necesario definir un conjunto de tipo de datos que definen las características de dichos. 


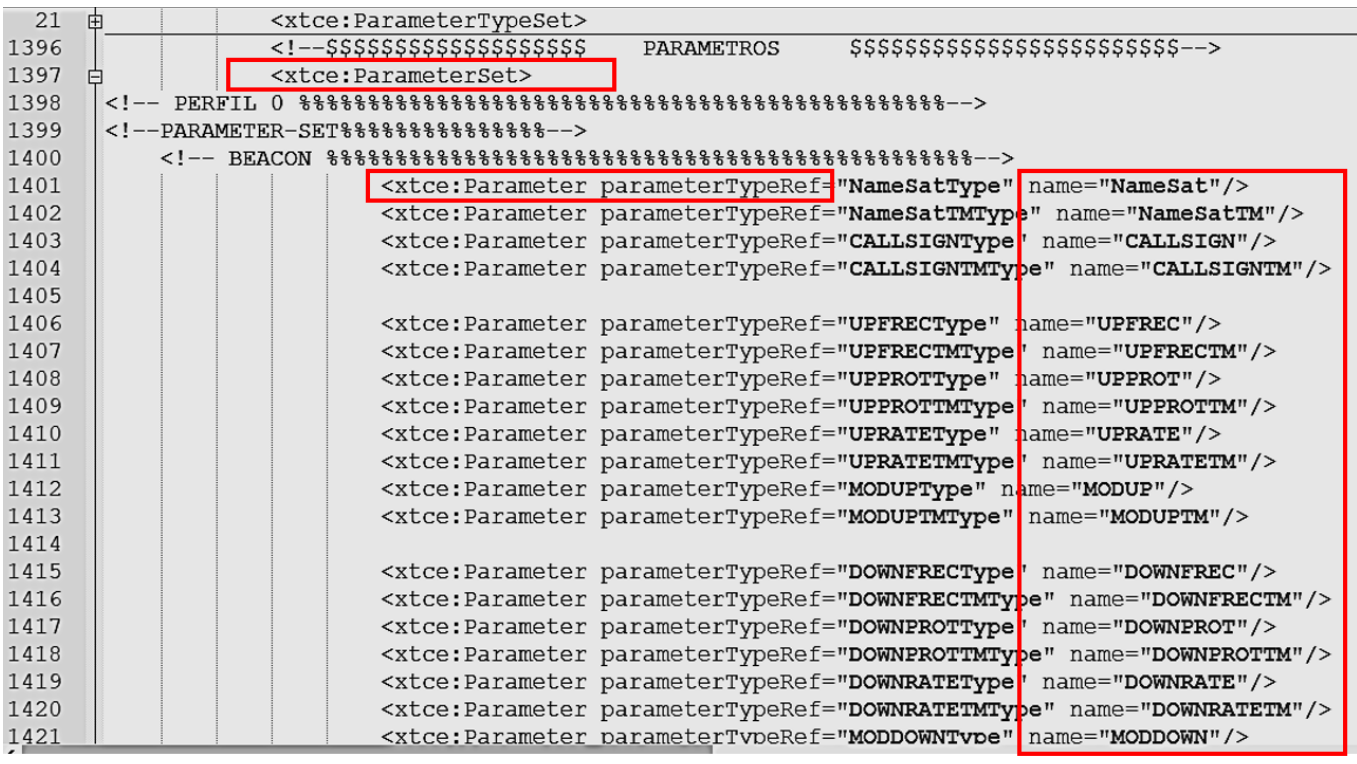

Figura 6. Descripción del conjunto de parámetros del perfil de radio faro.

Fuente: elaboración propia del autor.

De manera consecuente con los parámetros, tipos de parámetros y conjunto de datos deben ser referenciados a través de un conjunto de contenedores que asocian la información creada anteriormente. Los contenedores pueden ser establecidos a partir de categorías definidas en la estructura de datos del formato XTCE. En un caso práctico, dichos pueden hacer referencia a la información de voltajes, corrientes, temperaturas o el conjunto o todas ellas para los módulos y submódulos de misión, control de actitud, potencia, así como también periféricos del satélite mismo, componentes físicos, lógicos, disponibilidad de los sistemas de comunicación radio, controladores, parámetros de acceso, identificadores de posición, parámetros de temperatura global, estado de potencia general y cualquier otra información elemental que se considere como particular o general y que en un amplio sentido proporcione los datos necesarios para determinar el estado de salud de un componente en particular.

En la tabla 1 se muestra la descripción de perfiles y su respectiva asociación con el tipo de información de telemetría. En este punto es preciso reiterar que los perfiles de telemetría que se han diseñado como una ejemplificación de los contenedores de telemetría del estándar pueden ser descriptores de información de telemetría o, en su defecto, de información de telemetría. Por ejemplo: los perfiles de telemetría dos y tres contienen información relacionada con el tiempo y estado del sistema de sincronismo en el sistema satelital. Sin embargo, hay que precisar que el perfil de telemetría número dos posee los datos de tiempo y el tres contiene la información que describe el anterior perfil. En este contexto, hay dos que correlacionan un grupo de información en particular.

En la tabla 2 se observa cómo la información para el conjunto de datos de telemetría es agrupada en un perfil del sistema. En este también se observa que la información asociada al contenedor junta datos y metadatos de telemetría. En otros términos, este segmento contiene en un mismo paquete o trama de datos los componentes descriptivos necesarios para que la información de telemetría una vez recibida pueda ser interpretada de manera fácil y ágil a través de mecanismos de decodificación Ascii, pues este paquete de información es la metodología de presentación del sistema satelital ante cualquier estación terrena en recepción. 
Como se puede observar en la tabla 3, la parametrización de datos asociados al nombre del satélite puede o no contener tanta información como sea necesaria. En este ejemplo se muestra que el dato de telemetría "CUBESATUD" esta referenciado al dato "NameSat", que a su vez corresponde al tipo de parámetro "NameSatType" junto al contenedor "PERFILO" de la base de datos XTCE y que por su naturaleza una tiene categoría de tipo "string" el cual será codificado por el sistema en órbita como Ascii para su transmisión a través del radio faro ocupando una longitud máxima de "72" bits.

Tabla 1. Definición perfiles de telemetría

\begin{tabular}{cccccccc}
\hline Perfil & Información & Datos & Metadatos & Perfil & Información & Data & Metadatos \\
$\mathbf{0}$ & Beacon & $\mathrm{x}$ & $\mathrm{x}$ & $\mathbf{7}$ & TLE & & $\mathrm{x}$ \\
$\mathbf{1}$ & Perfiles & $\mathrm{x}$ & $\mathrm{x}$ & $\mathbf{8}$ & Voltajes & $\mathrm{x}$ & \\
$\mathbf{2}$ & Time & $\mathrm{x}$ & & $\mathbf{9}$ & Voltajes & & $\mathrm{x}$ \\
$\mathbf{3}$ & Time & & $\mathrm{x}$ & $\mathbf{1 0}$ & Corrientes & $\mathrm{x}$ & \\
$\mathbf{4}$ & Identificación & $\mathrm{x}$ & & $\mathbf{1 1}$ & Corrientes & & $\mathrm{x}$ \\
$\mathbf{5}$ & Identificación & & $\mathrm{x}$ & $\mathbf{1 2}$ & TEMP & $\mathrm{x}$ & \\
$\mathbf{6}$ & TLE & $\mathrm{x}$ & & $\mathbf{1 3}$ & TEMP & & $\mathrm{x}$ \\
\hline
\end{tabular}

Fuente: elaboración propia del autor.

Tabla 2. Estructura del perfil de telemetría, información del radio faro

\begin{tabular}{|c|c|c|c|c|c|}
\hline \multicolumn{3}{|c|}{ Beacon } & \multicolumn{3}{|c|}{ Beacon } \\
\hline Item & Dato/Metadato & Valor & Item & Dato/Metadato & Valor \\
\hline \multirow{3}{*}{1} & Dato & CUBESATUD & \multirow{3}{*}{6} & Dato & ASK \\
\hline & & & & & \\
\hline & Metadato & SATNAME,UTF8,,72, & & Metadato & UPMOD,UTF8,,32, \\
\hline \multirow{3}{*}{2} & Dato & QBUDC1 & \multirow{3}{*}{7} & Dato & 245015 \\
\hline & & & & & \\
\hline & Metadato & SSID,UTF8, 56, & & Metadato & DOWNFREC,IEEE754,KHZ,32, \\
\hline \multirow{3}{*}{3} & Dato & 144005 & \multirow{3}{*}{8} & Dato & CSP \\
\hline & & & & & \\
\hline & Metadato & UPFrec,IEEE754,KHZ,32, & & Metadato & DOWNPROT,UTF8,,24, \\
\hline \multirow{3}{*}{4} & Dato & AX25 & \multirow{3}{*}{9} & Dato & 9600 \\
\hline & & & & & \\
\hline & Metadato & UPPROT,UTF8,,32, & & Metadato & DOWNRATE,INT,baud,14, \\
\hline \multirow{2}{*}{5} & Dato & 9600 & \multirow{2}{*}{10} & Dato & FSK \\
\hline & Metadato & UPRATE,INT,baud,14, & & Metadato & DOWNMOD,UTF8,,32, \\
\hline
\end{tabular}

Fuente: elaboración propia del autor. 
Tabla 3. Parametrización de datos asociados al nombre del satélite, perfil 0.

\begin{tabular}{|c|c|c|c|c|}
\hline \multicolumn{5}{|c|}{ Beacon } \\
\hline Item & Dato/Metadato & Valor & Variable & Valor \\
\hline \multirow{6}{*}{1} & \multirow{6}{*}{ Dato } & \multirow{6}{*}{ CubeSatUD } & Tipo de dato & String \\
\hline & & & Nombre & NameSatType \\
\hline & & & Longitud & 72 \\
\hline & & & Unidad & N/A \\
\hline & & & Referencia & NameSat \\
\hline & & & Contenedor & Perfil 0 \\
\hline
\end{tabular}

Fuente: elaboración propia del autor.

\begin{tabular}{|c|c|c|c|c|c|c|}
\hline Container Name & Parameter Name & Parameter Alias(s) & Size & Start Bit & Value & $\mathrm{De}$ \\
\hline \multicolumn{6}{|l|}{ PROFILE01 } & \\
\hline PROFILE01 & NameSat & & 72 & 0 & $==$ CUBESATUD $\{\mathrm{cal}\}$ & \\
\hline PROFILE01 & CALLSIGN & & 48 & 72 & $==$ QBUDC $1\{\mathrm{cal}\}$ & \\
\hline PROFILE01 & UPFREC & & 32 & 120 & $==144.005\{\mathrm{cal}\}$ & \\
\hline PROFILE01 & UPPROT & & 32 & 152 & $==A X 25\{\mathrm{cal}\}$ & \\
\hline PROFILE01 & UPRATE & & 14 & 184 & $==9600\{\mathrm{cal}\}$ & \\
\hline PROFILE01 & MODUP & & 24 & 198 & $==\mathrm{ASK}\{\mathrm{cal}\}$ & \\
\hline PROFILE01 & DOWNFREC & & 32 & 222 & $==245.015\{\mathrm{cal}\}$ & \\
\hline PROFILE01 & DOWNPROT & & 24 & 254 & $==\operatorname{CSP}\{\mathrm{cal}\}$ & \\
\hline PROFILE01 & DOWNRATE & & 14 & 278 & $==9600\{\mathrm{cal}\}$ & \\
\hline PROFILE01 & MODDOWN & & 24 & 292 & $==\mathrm{FSK}\{\mathrm{cal}\}$ & \\
\hline PROFILE01 & NameSatTM & & 136 & 316 & $==$ SATNAME,UTF $8,, 72, \ldots$ & \\
\hline PROFILE01 & CALLSIGNTM & & 112 & 452 & $==S S I D, U T F 8,56,\{\mathrm{cal}\}$ & \\
\hline PROFILE01 & UPFRECTM & & 176 & 564 & $==$ UPFrec,IEEE $754, \mathrm{KH} . .$. & \\
\hline PROFILE01 & UPPROTTM & & 128 & 740 & $==$ UPPROT,UTF8 $, 32,\{\ldots$ & \\
\hline PROFILE01 & UPRATETM & & 152 & 868 & $==$ UPRATE,INT, baud, $1 \ldots$ & \\
\hline PROFILE01 & MODUPTM & & 120 & 1020 & $==U P M O D, U T F 8,, 32,\{\mathrm{c} \ldots$ & \\
\hline PROFILE01 & DOWNFRECTM & & 192 & 1140 & $==$ DOWNFREC,IEEE7 $\ldots$ & \\
\hline PROFILE01 & DOWNPROTTM & & 144 & 1332 & $==$ DOWNPROT,UTF ${ }_{,}, \ldots$ & \\
\hline PROFILE01 & DOWNRATETM & & 168 & 1476 & $==$ DOWNRATE,INT, ba... & \\
\hline PROFILE01 & MODDOWNTM & & 136 & 1644 & $==$ DOWNMOD,UTF8, $3 \ldots$ & \\
\hline
\end{tabular}

Figura 7. Validación y presentación de los datos en aplicación.

Fuente: elaboración propia del autor.

Para validación, verificación y simulación de la base de datos XTCE y su respectivo esquema de información se ha construido un compilador en Java que realiza la validación sintáctica en primera medida del esquema XML, seguido del esquema y formato XTCE. Posteriormente, con este mismo sistema se ha realizado un sistema de presentación que estructura toda la base de datos en tablas, resumiendo las características del sistema de datos y metadatos a ser transmitidos. La figura 7 muestra el resultado de la interfaz gráfica del sistema de validación y presentación de los datos ya expuestos. Este sistema permite obtener en una manera simple el esquema de información y realizar las correcciones o ajustes necesarios para diseñar la base de datos.

Seguido se ha utilizado la porción de código del sistema de telemetría para codificar la información acorde a los tipos de datos asignados a cada variable de medición. Este fragmento de código debe ser utilizado por el sistema en órbita para ejecutar las actividades recurrentes de codificación y almacenamiento de datos de telemetría. La figura 8 muestra el mensaje de salida; es decir, la codificación Ascii del perfil 0 para el sistema de telemetría que corresponde al 
contenedor uno y que será utilizado por la interfaz de comunicación del radio faro para informar a las estaciones terrenas la información del sistema satelital.

Para validar la salida codificada el programa de evaluación también ejecuta rutinas para decodificar un flujo de información y presentarla de manera estructurada y organizada. La figura 9 muestra la decodificación del flujo de datos recibido en estación terrena. De esta forma, este segmento de código corresponde al sistema de telemetría en tierra que debe ejecutar el proceso de decodificación una vez cuente con la base de datos de cada satélite, la cual puede ser descargada a través de los mismos medios empleando una codificación tacita (como lo es la codificación Ascii, empleada en la mayor parte del sistema para realizar notas textuales).

\section{Output Hexadecimal Binary}

0x43554245534154554457314c4d465231431001484158323596010dd40f5d0d4d425805341544e414d452c555446382c2c37322c535 349442 c555446382c2c35362c5550467265632c494545453735342cc4b485a2c33322c555050524f542c555446382c2c3332426175642c

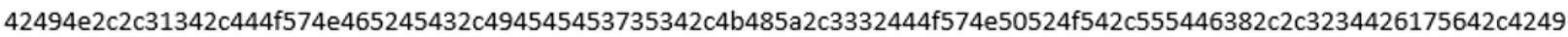
$4 \mathrm{e} 2 \mathrm{c} 2 \mathrm{c} 3134000$

Figura 8. Mensaje de salida, codificación Ascii del perfil 0 de telemetría.

Fuente: elaboración propia del autor.

\begin{tabular}{|c|c|c|c|c|}
\hline & $\begin{array}{l}\text { Container Contents } \\
\text { U/Calibrated Value ] [ Uncalit }\end{array}$ & rated Value ] [ Raw Value ] & & \\
\hline NameSat & CUBESATUD & CUBESATUD & $0 \times 435542455341545544$ & $\Delta$ \\
\hline CALLSIGN & W1LMFR1 & W1LMFR1 & $0 \times 57314 c 4 d 465231$ & \\
\hline UPFREC & 144.005 & 144.005 & $0 \times 43100148$ & \\
\hline UPPROT & AX25 & AX25 & $0 \times 41583235$ & \\
\hline UPRATE & 9600 & 9600 & $0 \times 2580$ & \\
\hline DOWNFREC & 245.015 & 245.015 & $0 \times 437503 d 7$ & \\
\hline DOWNPROT & CSP & $\operatorname{CSP}$ & $0 \times 435350$ & \\
\hline DOWNRATE & 9600 & 9600 & $0 \times 2580$ & \\
\hline NameSatTM & SATNAME,UTF8, 72 & SATNAME,UTF8, 72 & $0 \times 5341544 \mathrm{e} 414 \mathrm{~d} 452 \mathrm{c} 55 \mathrm{E}$ & \\
\hline CALLSIGNTM & SSID,UTF8,,56, & SSID,UTF8, 56 & $0 \times 535349442 c 55544638 \varepsilon$ & \\
\hline UPFRECTM & UPFrec,IEEE754,KHZ,32, & UPFrec,IEEE754,KHZ,32, & 0x5550467265632c4945 & \\
\hline UPPROTTM & UPPROT,UTF8,,32 & UPPROT,UTF8„,32 & $0 \times 555050524 f 542 c 55544$ & \\
\hline UPRATETM & Baud,BIN,,14, & Baud,BIN,,14, & $0 \times 426175642 \mathrm{c} 42494 \mathrm{e} 2 \mathrm{c} 2$ & \\
\hline DOWNFRECTM & DOWNFREC,IEEE754,KH & DOWNFREC,IEEE754,KH & $0 \times 444 f 574 \mathrm{e} 465245432 \mathrm{c} 4$ & \\
\hline DOWNPROTTM & DOWNPROT,UTF8,,24 & DOWNPROT,UTF8,,24 & $0 \times 444 f 574$ e $50524 f 542 c 5 E$ & \\
\hline DOWNRATETM & Baud,BIN,,14 & Baud,BIN,,14 & $0 \times 426175642 \mathrm{c} 42494 \mathrm{e} 2 \mathrm{c} 2$ & . \\
\hline
\end{tabular}

Figura 9. Decodificación del flujo de datos recibido en aplicación de estación terrena.

Fuente: elaboración propia del autor. 


\section{Conclusiones}

El marco referencial para la construcción de una base de datos para los nodos de un sistema satelital de carácter académico se consolida teniendo en cuenta la utilización de un estándar de carácter abierto y modular. El cual, además de ser una recomendación internacional para la construcción del sistema de telemetría y comando, proporciona un funcionalidad y aplicabilidad en entornos satelitales de tipo CubeSat.

La base de datos se ha construido y diseñado a partir de lenguajes de formato de datos XML y XTCE. También, se ha verificado su integridad mediante la codificación, decodificación y presentación a través de una interfaz gráfica construida en Java. De este modo, el concepto de una base de datos para el sistema de telemetría tanto de la estación terrena como del sistema en órbita proporcionará la afirmación de múltiples esfuerzos mediante arquitecturas distribuidas y verificables.

La consolidación del diseño de un sistema satelital, empleando una base de datos con estas características, ayuda a los desarrolladores a formalizar y ejemplificar sistemas satelitales para la puesta en marcha con herramientas de adopción progresiva para proyectos aeroespaciales que se encuentran en el proceso de participación en redes de estaciones terrenas.

\section{Agradecimientos}

Los investigadores resaltan la gestión colaborativa académica e investigativa de todos los docentes de las Universidad Distrital Francisco José de Caldas y de la Universidad Pedagógica y Tecnológica de Colombia, quienes aportaron en menor o mayor medida al desarrollo del proyecto.

\section{Referencias}

Alhammadi, M., Svetinovic, D. (2017). Autonomy requirements engineering for micro-satellite systems: CubeSat case study. En International Conference on
Information (hg.), XXVI International Conference on Information, Communication and Automation Technologies (s. 1-6). IEEE. https://doi.org/10.1109/ ICAT.2017.8171623

Anandan, V. K., Pradeep, K. C., Satyanarayana, S. N. V., Sarkar, M. (2018). Multiple Satellite Telemetry and Tracking System (MuST). En IEEE Indian Conference on Antennas and Propogation (InCAP) (s. 1-4). IEEE. https://doi.org/10.1109/INCAP.2018.8770889

Berthoud, L., Schenk, M. (2016). How to Set Up a CubeSat Project - Preliminary Survey Results. En 30th Annual AIAA/USU Conference on Small Satellites American Institute of Aeronautics and Astronautics Inc AIAA (AIAA).

Cappiello, A. G., Popescu, D. C., Harris, J. S., Popescu, O. (2019). Radio Link Design for CubeSat-to-Ground Station Communications Using an Experimental License. En ISSCS 2019: International Symposium on Signals, Circuits and Systems (s. 1-4). IEEE. https:// doi.org/10.1109/ISSCS.2019.8801767

Crisp, N. H., Smith, K., Hollingsworth, P. (2015). Launch and deployment of distributed small satellite systems. Acta Astronautica, 114, 65-78. https://doi.org/10.1016/j.actaastro.2015.04.015

Díaz, J. E. E., Hernández, A. A. R. (2015). Methodology to implement a satellite ground university station. En M. Fernández, G. Lefranc y R. Pérez (hg.), IEEE Chilean Conference on Electrical, Electronics Engineering, Information and Communication Technologies (pp. 261-267). Universidad Central de Chile; IEEE. https://doi.org/10.1109/Chilecon.2015.7400386

Díaz-González, F. A., Pinzón-Cabrera, P. R., Hernández-Calderón, C. M. (2016). Design of a Nanosatellite Ground Monitoring and Control Software - a Case Study. Journal of Aerospace Technology and Management, 8(2), 211-231. https://doi.org/10.5028/jatm.v8i2.553

Eatchel, A. L., Fevig, R., Cooper, C., Gruenenfelder, J., Wallace, J. (2015). Development of a Baseline Telemetry System for the Cubesat Program at the University of Arizona. International Telemetering Conference Proceedings. http://hdl.handle.net/10150/607488 
Electronics, Computing and Communication Technologies (IEEE Conecct) (2014). IEEE International Conference on. IEEE.

Espindola, J. (2017). An Autonomous and Dynamical Approach to Small Satellite Ground Stations Networks.

Felt, A. J. (2014). Federated ground station network model and interface specification. $\mathrm{Na}$ val Postgraduate School. https://calhoun.nps.edu/ handle/10945/44558

Fernandez, M. M., Rice, J. K., Smith, D., Jones, R. (2017). Sharing Telemetry across Organizations and Systems. En 6th IEEE International Conference on Space Mission Challenges for Information Technology (pp. 44-49). IEEE Computer Society, Conference Publishing Services. https://doi.org/10.1109/SMC-IT.2017.15

Kilic, C., Scholz, T., Asma, C. (2013). Deployment strategy study of QB50 network of CubeSats. En 6th International Conference on Recent Advances in Space Technologies (RAST) (pp. 935-939). IEEE. https://doi.org/10.1109/RAST.2013.6581349

Mishurov, A. V., Panko, S. P., Khnykin, A. V. (2017). Standards for the design of tracking, telemetry and command subsystem of spacecraft. En A. Kosykh (hg.), Dynamics of Systems, Mechanisms and Machines (pp. 1-4). IEEE. https://doi.org/10.1109/SIBCON.2017.7998441

Mision Operations Reference Model 1 (2010) i.d.F.v. Magenta Book.https://public.ccsds.org/publications/MagentaBooks.aspx

Miyazaki, Y., Yamazaki, M., Uehara, T., Tsunokawa, Y. (2020). Sprout Amateur Radio Slow Scan TV Satellite. Nihon en Tokio. http://sat.aero.cst.nihon-u. ac.jp/sprout-e/

Moreno, A., Sánchez, C. (2014). Diseño Del sistema de integración de subsistemas, análisis y pruebas del picosatélite Cubesat-Ud Colombia-1. Universidad Distrital Francisco José De Caldas.

Praks Jaan, J. N. (2016). Telecommand and Telemetry Implementation of Aalto-2 CubeSat Project. Space Science and Technology.

Ravichandran, P. N., Kulkarni, S., Sharma, S., Vasudevamurthy, H. S., Vanitha, M., Lakshminarsimhan, P.
(2009). Wireless Telecommand and Telemetry Systems for Satellite Communication Using ZigBee Network. En International Conference on Advances in Recent Technologies in Communication and Computing (pp. 274-278). IEEE. https://doi.org/10.1109/ ARTCom.2009.124

Scholz, A., Juang, J. N. (2015). Toward open source CubeSat design. Acta Astronautica, 115, 384-392. https://doi.org/10.1016/j.actaastro.2015.06.005

Shahu, K. (2018). Design of Telecommand Software and Packet Structure for Lunar Landing Mission. En Second International Conference on Electronics, Communication and Aerospace Technology (Iceca) (pp. 1641-1646). IEEE. https://doi.org/10.1109/ICECA.2018.8474845

Shamutally, F., Soreefan, Z., Suddhoo, A., Momple, J. M. (2018). Practical "Low Cost" LEO Receiving Ground Station. En IEEE Radio and Antenna Days of the Indian Ocean (Radio) (pp. 1-2). IEEE. https:// doi.org/10.23919/RADIO.2018.8572330

Shruthi, S., Pragathi, P., Shwetha, N., Swathi, S. (2017). CCSDS packet telemetry. En IEEE International Conference on Smart Technologies and Management for Computing, Communication, Controls, Energy and Materials: ICSTM (pp. 371-376). IEEE. https://doi.org/10.1109/ ICSTM.2017.8089187

Simon, G., Shaya, E., Rice, K., Cooper, S., Dunham, J., Champion, J. (2006). XTCE: a standard XML-schema for describing mission operations databases. En IEEE Aerospace Conference proceedings (pp. 3313-3325). IEEE. https://doi.org/10.1109/AERO.2004.1368138

Thiruppathirajan, S., Sheba Elizabeth, D., Preetha, C., Sreekumar, S., Vinod, P., Krishnan, A. R., Mookiah, T., Zachariah, J. P. (2014). An efficient secured CCSDS based telemetry system for ISRO's near earth and deep space missions. En IEEE International Conference on (pp. 1-6). IEEE. https://doi.org/10.1109/CONECCT.2014.6740175

Vera-Cely, O. F., Mesa-Lara, J. A., Ferro-Escobar, R. (2015). Análisis de velocidad de transmisión del protocolo AX.25 implementado en la estación terrena satelital UPTC. Revista Tecnura, 
19(46), 93. https://doi.org/10.14483/udistrital.jour. tecnura.2015.4.a07

Vertat, I., LInhart, R., Pokorny, M., Masopust, J., Fiala, P., Mraz, J. (2018). Small satellite ground station in Pilsen-experiences with VZLUSAT-1 commanding and future modifications toward open reference ground station solution. En 28th International Conference Radioelektronika (pp. 1-6). IEEE. https://doi.org/10.1109/ RADIOELEK.2018.8376393

White, D. J., Giannelos, I., Zissimatos, A., Kosmas, E., Papadeas, D. (2015). SatNOGS: Satellite Networked Open Ground Station. Engineering Faculty Publications., College of Engineering, 40, 40. 